\title{
Melatonin Promotes Myelination by Decreasing White Matter Inflammation After Neonatal Stroke
}

\author{
SONIA VILLAPOL, SÉBASTIEN FAU, SYLVAIN RENOLLEAU, VALÉRIE BIRAN, CHRISTIANE CHARRIAUT-MARLANGUE, \\ AND OLIVIER BAUD
}

INSERM AVENIR R05230HS (U676), Université Paris, Faculté de Médecine Denis Diderot, 75019 Paris, France

\begin{abstract}
Melatonin demonstrates neuroprotective properties in adult models of cerebral ischemia, acting as a potent antioxidant and anti-inflammatory agent. We investigated the effect of melatonin in a 7-d-old rat model of ischemia-reperfusion, leading to both cortical infarct and injury in the underlying white matter observed using MRI and immunohistochemistry. Melatonin was given i.p. as either a single dose before ischemia or a double-dose regimen, combining one before ischemia and one $24 \mathrm{~h}$ after reperfusion. At $48 \mathrm{~h}$ after injury, neither a significant reduction in cortical infarct volume nor a variation in the number of TUNEL- and nitrotyrosine-positive cells within the ipsilateral lesion was observed in melatonin-treated animals compared with controls. However, a decrease in the density of tomato lectin-positive cells after melatonin treatment was found in the white matter underlying cortical lesion. Furthermore, we showed a marked increase in the myelin basic protein-immunoreactivity in the cingulum and in the density of mature oligodendrocytes (APCimmunoreactive) in both the ipsilateral cingulum and external capsule. These results suggest that melatonin is not able to reduce cortical infarct volume in a neonatal stroke model but strongly reduces inflammation and promotes subsequent myelination in the white matter. (Pediatr Res 69: 51-55, 2011)
\end{abstract}

$\mathrm{N}$ eonatal hypoxia-ischemia is an important cause of neonatal brain injury, resulting in cerebral palsy, learning disabilities, visual field deficits, and epilepsy (1). In addition to global cerebral ischemia arising from systemic asphyxia, recent data suggest a higher incidence of focal ischemiareperfusion leading to stroke in near-term neonates $(2,3)$. Mechanisms of arterial ischemic injury without the confounding effect of hypoxia are not fully understood. Although therapeutic hypothermia has recently demonstrated a benefit by decreasing brain tissue injury in infants with hypoxicischemic encephalopathy $(4,5)$, very little is known regarding potential neuroprotective strategies after neonatal stroke.

Melatonin, an indoleamine, is synthesized and secreted from the pineal gland relative to the circadian rhythms. Melatonin readily crosses the blood-brain barrier, and after exogenous administration, it is found in high concentrations in the brain. It was demonstrated that melatonin has neuroprotective effects through either antiapoptotic (6) or antioxidant effects $(7,8)$ or by decreasing the excitotoxic cascade $(9)$. Melatonin

Received May 24, 2010; accepted August 10, 2010

Correspondence: Christiane Charriaut-Marlangue, Ph.D., INSERM AVENIR R05230HS (U676), Hôpital Robert Debré, 48 Bd Sérurier, 75019 Paris, France; e-mail: christiane.marlangue@gmail.com.

Supported by funds from "Mairie de Paris" and "PremUp fundation."

C.C.-M. and O.B. contributed equally to this work. has demonstrated neuroprotective properties in adult models of cerebral ischemia, acting as a potent antioxidant and antiinflammatory agent $(10,11)$. Melatonin was shown to have a potent protective effect in several models of developing white matter damage, by not only promoting oligodendroglial maturation and myelination repair but also decreasing astrogliosis and microglial activation (12-15). We previously developed a neonatal model of stroke in 7-d-old (P7) rat characterized by apoptotic cell death $(16,17)$, inflammatory responses $(18,19)$, and oxidative stress (20). In addition to cerebral infarct, this model induced glial activation in underlying white matter including persistent activation of microglia and astrogliosis $(19,21)$. As the presence of activated microglia is known to impair the oligodendroglial lineage, we hypothesized that melatonin could be used as an effective neuroprotectant in this stroke model.

In summary, in this study, we show that melatonin, despite no effect on stroke lesion size, has a potent protective effect on white matter damage in P7 animals subjected to left middle cerebral artery electrocoagulation followed by a transient bilateral common carotid artery occlusion. Melatonin seems to act by promoting oligodendroglial maturation and myelination repair, together with modulation of microglial activation.

\section{MATERIALS AND METHODS}

Neonatal ischemia. Full details of the study have been approved by Robert Debré Research council review board; the approval number is 2009-02. All experiments were performed in compliance with the ethical rules of INSERM. Ischemia was performed on Wistar 7-d-old rat pups (17-21 g; Janvier, Le Genest St-Isle, France) of both sexes. Briefly, anesthetized (with isoflurane, $1.5 \%$ for induction and $1 \%$ for maintenance) rats were exposed to left middle cerebral artery electrocoagulation followed by a 50 -min both common carotid arteries occlusion. During ischemia and the first hour of recovery, the pups were placed in a humidified incubator at $35^{\circ} \mathrm{C}$ and then transferred to their mothers. Lesions were monitored at $48 \mathrm{~h}$ using a scale from 0 to 3 , where 0 indicated no observable lesion and 1,2, and 3 indicated small, medium, and large infarct, respectively, as previously described (22).

Melatonin treatment. Melatonin (Sigma Chemical Co. Aldrich) was dissolved in $1 \mathrm{~mL}$ of normal saline solution containing $<5 \%$ DMSO. Animals subjected to the ischemia-reperfusion procedure $(n=36)$ were randomly assigned to one of the three following groups: 1) control group receiving saline buffer (vehicle-treated animals, $n=12$ ), 2) melatonin-treated group receiving a single dose of melatonin $(20 \mathrm{mg} / \mathrm{kg})$ or its vehicle given via an i.p. injection $1 \mathrm{~h}$ before the onset of ischemia (Mel-1dose, $n=12$ ), and 3 ) melatonin-treated group receiving a double dose of melatonin $1 \mathrm{~h}$ before and $24 \mathrm{~h}$ after ischemia (Mel-2doses, $n=12$ ). Four animals were excluded from

\footnotetext{
Abbreviations: ADC, apparent diffusion coefficient; GFAP, glial fibrillary acidic protein; MBP, myelin basic protein; P7, postnatal day 7; $\mathbf{T L}$, tomato lectin
} 
the analysis: one dead in the two groups with melatonin-treated animals, and two were excluded because of the presence of a severe iatrogenic hemorrhage in vehicle-treated animals.

MRI acquisition. Rat pups were imaged during ischemia and reperfusion and at $48 \mathrm{~h}$. For imaging, pups were anesthetized by isoflurane $0.8 \%$ mixed in $30 \% \mathrm{O}_{2}, 70 \% \mathrm{~N}_{2}$ and thermoregulated by a water blanket heated at $37^{\circ} \mathrm{C}$. Images were acquired using a 7-T horizontal MRI device (Bruker, Germany). Apparent diffusion coefficient (ADC) maps were obtained from an EPI sequence $\left(\mathrm{TE}=25 \mathrm{~ms}, \mathrm{TR}=3 \mathrm{~s}, b\right.$ values $=50,500,1000 \mathrm{~s} / \mathrm{mm}^{2}$, FOV $40 \times 40 \mathrm{~mm}$, matrix $256 \times 256$, repetitions $=3,25$ slices, thickness $0.5 \mathrm{~mm}$, $9.5 \mathrm{~min})$. T2-weighted imaging was obtained with a RARE sequence (TR $=$ $5404 \mathrm{~ms}$, TE values $=25.6,76.8,128,179.2 \mathrm{~ms}$, RARE factor $=4)$. ADC maps were defined by applying a threshold of [mean - 2SD] of the whole contralateral hemisphere ADC.

Measurement of infarct volume. Rat pups were killed at $48 \mathrm{~h}$. Brains were removed, fixed for $5 \mathrm{~d}$ in $4 \%$ formol, and then embedded in paraffin and cut into $20-\mu \mathrm{m}$-thick sections. Sixteen sections from anterior striatum to posterior hippocampus were selected, taken at equally spaced 0.5 -mm intervals. Infarct size was determined on cresyl violet-stained sections using an image analyzer (ImageJ, National Institutes of Health, MA, http://rsb.info.nih.gov/ij/). Infarct volumes were expressed as the percentage of the ipsilateral hemisphere.

Immunohistochemistry and TUNEL staining. Sections (from vehicleand melatonin two doses-treated animals) were processed for endogenous peroxidase inactivation and blocked in $0.05 \mathrm{M}$ Tris-buffered saline (TBS), containing $10 \%$ FCS and $1 \%$ of Triton X-100 for $1 \mathrm{~h}$. Afterward, they were incubated overnight at $4{ }^{\circ} \mathrm{C}$ with either one of the following primary antibodies: tomato lectin (TL; 1:500, ABCYS, Paris, France), nitrotyrosine (NT: 1:100, Millipore, St-Quentin-en-Yvelines, France), Glial fibrillary acidic protein (GFAP, 1:500, ABCYS, Paris, France), APC (1:500, Calbiochem, StQuentin-en-Yvelines, France), and myelin basic protein (MBP, 1:1000, Millipore, St-Quentin-en-Yvelines, France). After several washings, sections were incubated for $2 \mathrm{~h}$ with biotinylated anti-rabbit or anti-mouse secondary antibody, respectively. Immunolabeling with the primary antibodies was visualized using the streptavidin-biotin-peroxydase method, as previously described (23). Adjacent sections were also processed for TUNEL staining according to the manufacturer's instructions (Roche, Meylan, France).

Cell counting and measurements of MBP-immunoreactivity. Immunoreactive cells were counted in the ipsilateral and contralateral cingulum and external capsule in at least nine animals per group under an $40 \times$ objective using a $0.065-\mathrm{mm}^{2}$-grid in three to four coronal sections for each animal at $48 \mathrm{~h}$ of recovery. Quantitative analysis of the optical density of MBPimmunoreactive fibers was done using image-analysis software (ImageJ, National Institutes of Health) as previously described (13). Nonspecific background density was measured at each brain level in an area devoid of MBP immunostaining and subtracted from the values for the cingulum.

Statistical analysis. Infarct score and volumes are expressed as the mean \pm SD and cell counts as the mean \pm SEM. Values were analyzed by one-way ANOVA with post hoc Bonferroni test and with the nonparametric Mann-Whitney test. Statistical analysis was performed using GraphPad Prism version 5.00 for Windows (GraphPad Software, San Diego, CA, www.graphpad.com).

\section{RESULTS}

\section{Microglial activation associated with MR abnormalities in} the underlying white matter after neonatal stroke. Using MRI ADC maps and T2-weighted imaging, we observed a cortical lesion as early as $60 \mathrm{~min}$ after the ischemic onset (Fig. 1A) well delineated on T2W images $6 \mathrm{~h}$ later (Fig. 1B). The extent of the cortical lesion was similar on cresyl violetstained sections and T2W images at 48 -h postlesion (Fig. $1 C$ and $D$ ). Interestingly, we also detected a spread of T2 abnormalities toward the subcortical white matter (indicated by arrows in Fig. 1D). At this time point, increased microglia activation was observed both in the different cortical layers and underlying white matter (Fig. 1E). Because melatonin is recognized to combine antioxidant and anti-inflammatory properties $(13,24)$, we asked the question whether this molecule could induce neuroprotection of the injured cortex and/or white matter by modulating this inflammatory response.

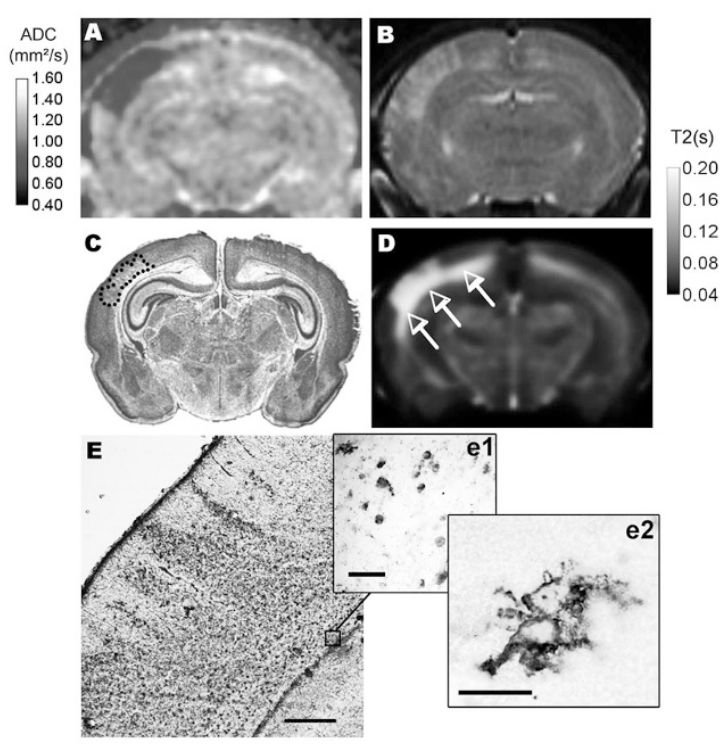

Figure 1. Time-course of the lesion and microglial activation after ischemia in $\mathrm{P} 7$ rat. (A) ADC map at 60 min after the ischemic onset showing a cortical lesion. $(B) \mathrm{T} 2$-weighted image at $6 \mathrm{~h}$ after ischemia demonstrating the cortical lesion displaying columnar aspects. $(C)$ Cresyl violet-stained sections at $48 \mathrm{~h}$ showing a cortical lesion (delineated with dots). (D) T2-weighted image at $48 \mathrm{~h}$ showing a spread toward subcortical and white matter (white arrows). (E) Microglia recruitment (TL immunostaining) both in the cortex (note columnar aspects) and the subcortical white matter (e1, enlarged panel; e2, microglial morphology). Images $(A),(B)$, and $(D)$ were obtained with the use of a high-resolution matrix $(0.014 \times 0.014 \mathrm{~mm} / \mathrm{pixel})$. Scale bar represents $200 \mu \mathrm{m}(E), 50 \mu \mathrm{m}(\mathrm{e} 1), 20 \mu \mathrm{m}(\mathrm{e} 2)$.
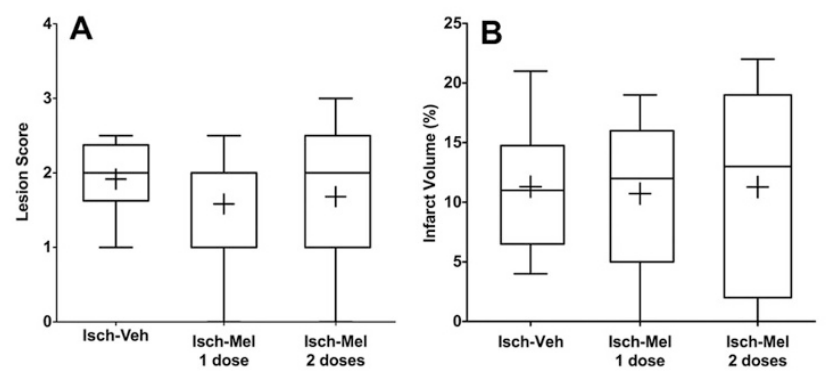

Figure 2. Effect of melatonin after neonatal stroke. (A) Melatonin did not induce a significant reduction of the lesion score in the Isch-Veh $(n=10)$, Isch-Mel-1-dose $(n=11)$, and Isch-Mel-2-doses $(n=11)$ groups. The median value for all groups was 2 and the mean $(+)$ was 1.9, 1.7, and 1.6, respectively. $(B)$ Percentage of infarct volume with respect to the total volume of the ipsilateral hemisphere in the Isch-Veh $(n=10)$, Isch-Mel-1-dose $(n=$ 11 ), and Isch-Mel-2-doses $(n=11)$ groups. The median (horizontal bar) was 11,13 , and 12 and the mean (+) was 11.3, 11.3 and 10.7, respectively. Data are expressed as the mean $\pm \mathrm{SD}$.

Melatonin did not significantly reduce cortical ischemic lesion volume. Two different regimens of melatonin treatment were used. A first dose of melatonin $(20 \mathrm{mg} / \mathrm{kg}$, i.p.) was given immediately before the onset of ischemia and did not affect the extent of brain damage evaluated $48 \mathrm{~h}$ after reperfusion (mean score $1.7 \pm 0.7, n=11$ ) compared with vehicle-treated animals $(1.9 \pm 0.5, n=10$; Fig. $2 A)$. In the double-dose regimen group, a second dose of melatonin was given $24 \mathrm{~h}$ after the first one. This treatment still did not reduce the lesion score (mean score $1.6 \pm 0.8, n=11$, Fig. 


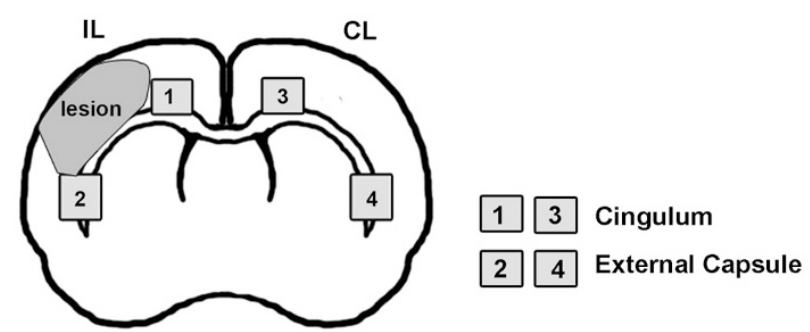

Figure 3. An illustration of cerebral hemispheres showing the lesion (gray) and counting areas: square 1 and 3 corresponding to cingulum, and square 2 and 4 to the external capsule, in the ipsilateral (IL) and contralateral (CL) hemispheres, respectively.

Table 1. Quantification of the number of TUNEL-, Ki67-, TL-, GFAP-, and NT-positive cells in the vehicle-treated $(n=10)$ and Isch-Mel $(n=11)$ groups in the IL and CL hemispheres

\begin{tabular}{lcccc}
\hline & Veh-IL & Mel-IL & Veh-CL & Mel-CL \\
\hline TUNEL & & & & \\
$\quad$ Cingulum & $2.9 \pm 0.3$ & $2.7 \pm 0.5$ & $1.9 \pm 0.5$ & $2.4 \pm 0.5$ \\
$\quad$ External capsule & $4.2 \pm 1.2$ & $5.1 \pm 1.3$ & $2.0 \pm 0.6$ & $1.1 \pm 0.4$ \\
Ki67 & & & & \\
$\quad$ Cingulum & $24.8 \pm 3.6$ & $32.3 \pm 2.5^{*}$ & $6.6 \pm 1.3$ & $11.6 \pm 1.6^{*}$ \\
$\quad$ External capsule & $26.4 \pm 3.3$ & $24.4 \pm 3.8$ & $3.8 \pm 2.7$ & $5.4 \pm 1.2$ \\
TL & & & & \\
$\quad$ Cingulum & $25.7 \pm 1.5$ & $18.1 \pm 2.4 \dagger$ & $14.8 \pm 1.8$ & $11.0 \pm 1.4$ \\
$\quad$ External capsule & $22.8 \pm 1.3$ & $13.8 \pm 1.4 \dagger$ & $12.8 \pm 1.5$ & $11.8 \pm 0.6$ \\
GFAP & & & & \\
$\quad$ Cingulum & $37.5 \pm 5.7$ & $44.5 \pm 9.0$ & $14.5 \pm 5.5$ & $16.0 \pm 6.9$ \\
$\quad$ External capsule & $21.7 \pm 4.1$ & $25.5 \pm 2.3$ & $11.2 \pm 4.3$ & $11.5 \pm 5.6$ \\
NT & & & & \\
$\quad$ Cingulum & $19.3 \pm 3.6$ & $18.1 \pm 2.9$ & $12.7 \pm 3.0$ & $13.1 \pm 2.5$ \\
$\quad$ External capsule & $16.1 \pm 2.6$ & $19.7 \pm 1.0$ & $11.0 \pm 2.6$ & $13.8 \pm 1.8$ \\
\hline
\end{tabular}

Note that melatonin (two doses) induced a significantly smaller number of TL-positive cells in the IL white matter at 48 -h postischemia. Data are given in mean \pm SEM positive cells $/ 0.065 \mathrm{~mm}^{2}$.

$* p<0.05$ (Mann-Whitney test).

$\dagger p<0.05$ (Bonferroni test).
$2 A)$. Furthermore, the melatonin treatment did not significantly affect the proportion of cortical infarct volume with respect to the total ipsilateral hemisphere volume (vehicletreated animals, $11.3 \pm 5.6 \%$; Mel-1dose, $11.3 \pm 8.3 \%$; and Mel-2doses, $10.7 \pm 6.3 \%$, mean \pm SD; Fig. $2 B$ ).

Melatonin reduced inflammation in the underlying white matter after ischemia-reperfusion. We next analyzed the white matter underlying cortical stroke in both the cingulum and external capsule (Fig. 3), where cell damage has been previously reported (19). Melatonin treatment was associated with a significant decrease in the density of TL-positive cells in the cingulum (by $30 \%, p<0.05$ ) and external capsule (by $40 \%, p<0.05)$ when compared with vehicle-treated animals (Table 1). In contrast, no significant difference in the density of TUNEL + cells after melatonin treatment was detected within these areas. Similarly, neither oxidative stress highlighted by 3-NT immunostaining nor astrogliosis detected using GFAP staining demonstrates a significant difference between vehicle- and melatonin-treated animals. Interestingly, melatonin treatment was associated with an increased proliferative cell density (Ki67 + cells) in both contralateral $(p<$ $0.02)$ and ipsilateral $(p<0.04)$ hemispheres (Table 1).

Melatonin treatment increases the number of myelinating fibers and mature oligodendrocytes. As melatonin was very recently reported to promote oligodendroglial maturation in antenatal hypoxia-induced white matter injury (13), we evaluated its effect on MBP fibers density and oligodendrocyte maturation after neonatal stroke in P7 rats. Compared with P9 controls, MBP-positive fibers density was significantly diminished in both the cingulum and external capsule beneath the cortical lesion observed in ischemic animals (Fig. 4A-E). In melatonin-treated animals, MBP-positive fibers density increased by $26 \%(p<0.05)$ in the cingulate white matter compared with vehicle-treated rat pups (Fig. $4 A, B$, and $E$ ). A similar trend was found in increased MBP-immunopositive

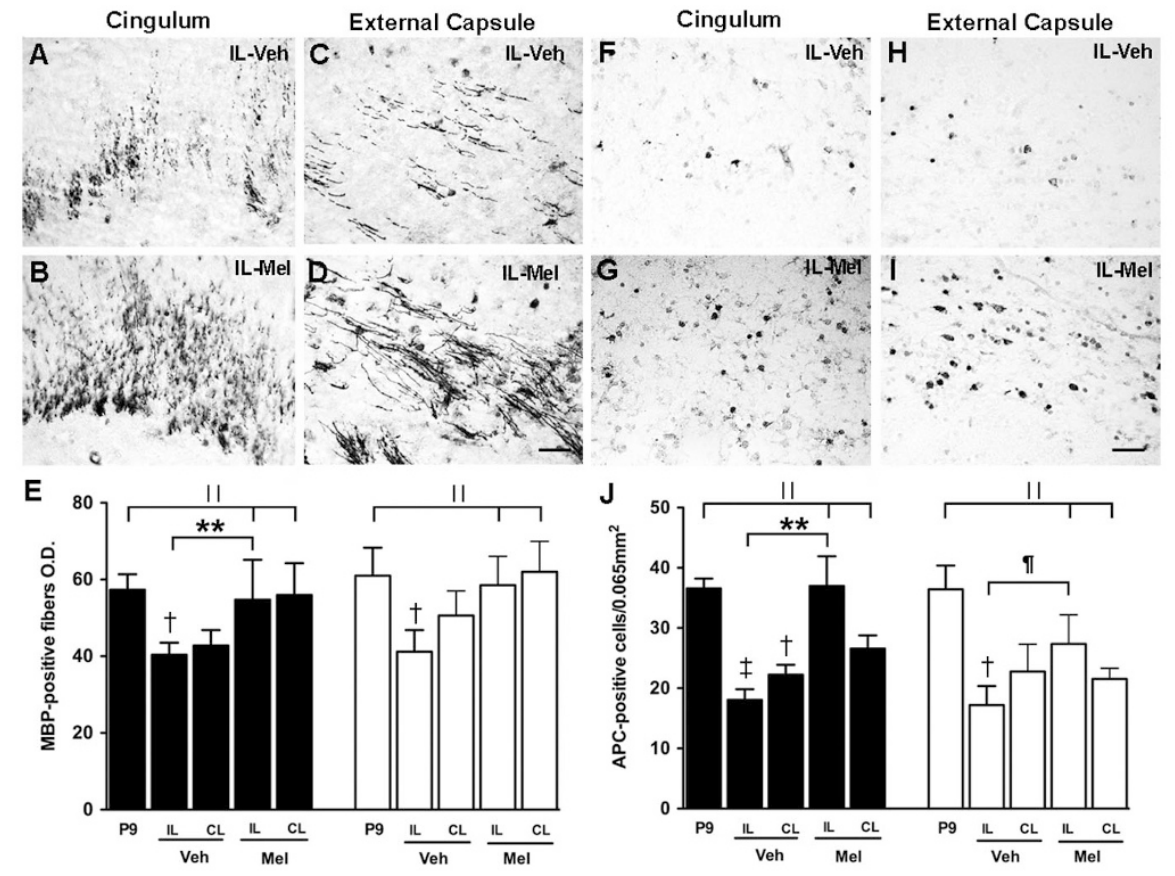

Figure 4. Melatonin (two doses) attenuates the myelination defect and increases the number of mature oligodendrocytes in the cingulum and external capsule at $48 \mathrm{~h}$ after ischemia-reperfusion. MBPimmunoreactive fibers density was found increased in melatonin-treated animals $(B)$ compared with vehicle-treated animals $(A)$ in the cingulum. In contrast, no significant change in MBP-immunoreactive fibers density was observed in the external capsule after melatonin treatment $(C-D)$. Scale bar represents 50 $\mu \mathrm{m}$. (E) Quantitative analysis of the optical density of MBP staining in the cingulate white matter and external capsule within vehicle- and melatonin-treated animals. ** $p<0.01 ; \dagger p<0.05 ; \ddagger p<0.01$ vs $\mathrm{P} 9$ controls. The number of APC-positive cells was found increased in melatonin-treated animals $(G-I)$ compared with vehicle-treated animals $(F-H)$ both in the cingulum and external capsule, respectively. $(J)$ Quantitative analysis of APC-positive cells density in the cingulate white matter and external capsule from vehicle- $(n=10)$ and melatonin-treated $(n=11)$ animals in IL hemisphere. Melatonin significantly increased the density of mature oligodendrocytes. Data are given in mean \pm SEM positive cells/ $/ 0.065$ $\mathrm{mm}^{2}$. $\|$, nonsignificant. 
fibers density after melatonin treatment in the external capsule (Fig. $4 C, D$, and $E$ ). Similar results were obtained with the two different regimens of melatonin treatment used.

Compared with P9 controls, the mature oligodendrocytes $(\mathrm{APC}+)$ density was found dramatically decreased in both cingulum and external capsule underlying cortical lesion in ischemic animals (Fig. $4 F$ and $H$ ). A higher density of mature oligodendrocytes $(\mathrm{APC}+)$ in the melatonin-treated animals was observed in both the cingular white matter (by $51 \%, p<$ 0.01 ) and external capsule (by $37 \%, p<0.05$; Fig. $4 F-J$ ). Interestingly, melatonin treatment had no effect on the contralateral white matter injury, suggesting that melatonin was able to promote oligodendroglial maturation only during cell proliferation and/or microglial activation (see Fig. 3).

\section{DISCUSSION}

In the neonatal stroke model used in this study, an inflammatory response of the developing white matter occurred between 24 and $72 \mathrm{~h}$ after ischemia-reperfusion procedure, at a developmental stage (P7) of high vulnerability to selective white matter injury (19). We have previously described in this model that cortical damage was also accompanied by a marked MBP reduction, O4+ oligodendrocytes depletion (19), and astrocyte demise (21) in subcortical white matter. In this study, we show that melatonin was not able to reduce infarct volume but was potently able to prevent the reduction of myelin content observed within the white matter underlying cortical lesion. This effect was associated with enhanced oligodendroglial maturation and reduced microglial activation.

Endogenous melatonin was found protective (9), and there is compelling in vivo and in vitro evidence supporting the use of exogenous melatonin to protect against focal and global ischemia-reperfusion injury in adult animals (25-28). Pretreatment with a single dose significantly reduces the infarct volume by $\sim 40 \%$ without affecting the hemodynamic parameters and regional cerebral blood flow (10). Melatonin decreases infarct size and improves neurological scores after middle cerebral artery occlusion in adult mice (29). In contrast, little is known about the effect of melatonin in neonatal hypoxic-ischemic animal models; two recent studies have suggested that melatonin could have beneficial effects and long-term neuroprotection (30) by reducing oxidative stress (31). Here, we refined these findings using a rat model of neonatal stroke, a model recognized to be of particular relevance in the setting of human neonatal stroke.

First, we tested the ability of melatonin to reduce cortical lesion induced in our stroke model administered through one or two dose regimen before ischemic damage. We hypothesized that melatonin could be a potential candidate for cortical neuroprotection, thanks to its general properties on oxidative stress and cell death. Indeed, melatonin reduces oxidative damage generated during both ischemia and reperfusion in neonatal rats and preserves mitochondrial function by improving the electron transport chain, maintaining ATP production, and restoring glutathione levels (32). Moreover, melatonin modulates reactive nitrogen species accumulation by inhibit- ing NO synthases (33), scavenging NO, and decreasing the production of the peroxynitrite (33). In addition, melatonin can increase the antioxidative activities of superoxide dismutase (SOD) enzymes and glutathione-peroxidase (GSH-Px) in fetal rats (34). Despite these properties, we failed to observe any effect of melatonin on cortical lesion size, suggesting that either the melatonin target might not be located in the cortical plate or cortical injury may be too severe to be reversible. Accordingly, melatonin was found unable to reduce cell death and protein nitration induced by ischemia-reperfusion.

Microglia infiltration occurs over several days in the cingulum in our stroke model (19), similarly to the one observed in the acute stage of periventricular leukomalacia's pathogenesis (35). Melatonin has been demonstrated to have potent neuroprotective effect on neonatal damage mimicking lesions of the developing white matter observed in preterm humans $(13,36-$ 39). On the basis of these properties, we then focused on the potential effect of melatonin on white matter damage. Melatonin treatment reduced microglial activation and subsequently enhanced oligodendroglial maturation and myelination of cingulate white matter and external capsule. A reduction in the number of activated microglia cells in the white matter was also found in mid-gestation fetal sheep after umbilical cord occlusion and melatonin treatment (37). Similar to a previous study using a rat model of white matter damage after antenatal hypoxia-ischemia (13), melatonin has, in our model, a protective effect on ipsilateral damaged white matter through decrease of microglial activation and maintain of oligodendroglial maturation leading to a normalization of the myelination process. To date, it has been shown that the effect of melatonin include endocrine, autocrine, and paracrine actions, decreased inflammation, and antioxidant effects. However, in most of perinatal brain damage animal models reported, immune cell activation frequently occurs (35) and melatonin effect seems to be closely linked to inflammation modulation.

It is relevant to point out that the melatonin-induced preservation of myelination is associated with a significant increased of postmitotic oligodendrocytes (APC-positive cells). It was suggested that the main target of melatonin treatment could be the maturation process of the oligodendrocyte lineage (13). Conversely, assuming that the cingulate myelination deficit was associated with focal cingulate microglial activation in our stroke model, and that immature oligodendrocytes are highly vulnerable in this inflammatory context (19) as, it is reasonable to hypothesize that melatonin could restore normal oligodendrocyte functions through a reduction in white matter inflammation. Surprisingly, the effect of melatonin seems to be restricted to ipsilateral injured white matter and not found in the contralateral hemisphere. In contrast, melatonin had no effect on the reactive astrocytes density, suggesting that both microglial cells and oligodendrocytes may be the only two cell types targeted by melatonin in the developing white matter.

Finally, as previously reported in a rat model of specific white matter injury (13), we found that melatonin was able to induce cell proliferation in our stroke model. Because cell proliferation was found particularly important in the ipsilateral 
lesioned hemisphere, we hypothesize that it may potentiate the effect of melatonin leading to white matter repair.

In conclusion, we highlight the potential therapeutic value of melatonin in specifically preventing white matter injury associated with neonatal stroke. In addition, our results reinforce the relationship between myelination and inflammation. It is now clear from published clinical trials that induction of hypothermia in infants who had perinatal asphyxia results in improved neurologic outcomes in survivors (40). In contrast, no neuroprotective strategy is available in the setting of neonatal stroke. Melatonin treatment might be used in highrisk pregnancies where ischemic events are common, or, because melatonin is a very safe pharmacological agent without side effects, in the hour before cesarean delivery in patients having variable or late decelerations (indications of ischemic risk). In addition, in newborn infants suffered from a stroke (with a diagnosis of seizures) melatonin could be early administered to at least reduce white matter damage.

Acknowledgment. We thank Dr. Todd James Treangen for revising the manuscript.

\section{REFERENCES}

1. Ferriero DM 2004 Neonatal brain injury. N Engl J Med 351:1985-1995

2. Golomb MR, Garg BP, Saha C, Azzouz F, Williams LS 2008 Cerebral palsy after perinatal arterial ischemic stroke. J Child Neurol 23:279-286

3. Lynch JK, Hirtz DG, DeVeber G, Nelson KB 2002 Report of the National Institute of Neurological Disorders and Stroke workshop on perinatal and childhood stroke. Pediatrics 109:116-123

4. Rutherford M, Ramenghi LA, Edwards AD, Brocklehurst P, Halliday H, Levene M, Strohm B, Thoresen M, Whitelaw A, Azzopardi D 2010 Assessment of brain tissue injury after moderate hypothermia in neonates with hypoxic-ischaemic encephalopathy: a nested substudy of a randomised controlled trial. Lancet Neurol 9:39-45

5. Jacobs S, Hunt R, Tarnow-Mordi W, Inder T, Davis P 2007 Cooling for newborns with hypoxic ischaemic encephalopathy. Cochrane Database Syst Rev CD003311

6. Kilic U, Kilic E, Lingor P, Yulug B, Bahr M 2004 Rifampicin inhibits neurodegeneration in the optic nerve transection model in vivo and after 1-methyl-4phenylpyridinium intoxication in vitro. Acta Neuropathol 108:65-68

7. Tütüncüler F, Eskiocak S, Basaran UN, Ekuklu G, Ayvaz S, Vatansever U 2005 The protective role of melatonin in experimental hypoxic brain damage. Pediatr Int 47:434-439

8. Cuzzocrea S, Costantino G, Gitto E, Mazzon E, Fulia F, Serraino I, Cordaro S, Barberi I, De Sarro A, Caputi AP 2000 Protective effects of melatonin in ischemic brain injury. J Pineal Res 29:217-227

9. Manev H, Uz T, Kharlamov A, Joo JY 1996 Increased brain damage after stroke or excitotoxic seizures in melatonin-deficient rats. FASEB J 10:1546-1551

10. Pei Z, Ho HT, Cheung RT 2002 Pre-treatment with melatonin reduces volume of cerebral infarction in a permanent middle cerebral artery occlusion stroke model in the rat. Neurosci Lett 318:141-144

11. Pei Z, Cheung RT 2004 Pretreatment with melatonin exerts anti-inflammatory effects against ischemia/reperfusion injury in a rat middle cerebral artery occlusion stroke model. J Pineal Res 37:85-91

12. Hutton LC, Abbass M, Dickinson H, Ireland Z, Walker DW 2009 Neuroprotective properties of melatonin in a model of birth asphyxia in the spiny mouse (Acomys cahirinus). Dev Neurosci 31:437-451

13. Olivier P, Fontaine RH, Loron G, Van Steenwinckel J, Biran V, Massonneau V, Kaindl A, Dalous J, Charriaut-Marlangue C, Aigrot MS, Pansiot J, Verney C, Gressens P, Baud O 2009 Melatonin promotes oligodendroglial maturation of injured white matter in neonatal rats. PLoS ONE 4:e7128

14. Hamada F, Watanabe K, Wakatsuki A, Nagai R, Shinohara K, Hayashi Y, Imamura R, Fukaya T 2010 Therapeutic effects of maternal melatonin administration on ischemia/reperfusion-induced oxidative cerebral damage in neonatal rats. Neonatology $98: 33-40$

15. Kaur C, Sivakumar V, Ling EA 2010 Melatonin protects periventricular white matter from damage due to hypoxia. J Pineal Res 48:185-193
16. Renolleau S, Aggoun-Zouaoui D, Ben-Ari Y, Charriaut-Marlangue C 1998 A mode of transient unilateral focal ischemia with reperfusion in the P7 neonatal rat: morphological changes indicative of apoptosis. Stroke 29:1454-1460; discussion 1461

17. Renolleau S, Fau S, Goyenvalle C, Joly LM, Chauvier D, Jacotot E, Mariani J, Charriaut-Marlangue C 2007 Specific caspase inhibitor Q-VD-OPh prevents neonatal stroke in P7 rat: a role for gender. J Neurochem 100:1062-1071

18. Benjelloun N, Renolleau S, Represa A, Ben-Ari Y, Charriaut-Marlangue C 1999 Inflammatory responses in the cerebral cortex after ischemia in the P7 neonatal Rat. Stroke 30:1916-1923; discussion 1923

19. Biran V, Joly LM, Heron A, Vernet A, Vega C, Mariani J, Renolleau S, CharriautMarlangue C 2006 Glial activation in white matter following ischemia in the neonatal P7 rat brain. Exp Neurol 199:103-112

20. Coeroli L, Renolleau S, Arnaud S, Plotkine D, Cachin N, Plotkine M, Ben-Ari Y, Charriaut-Marlangue C 1998 Nitric oxide production and perivascular tyrosine nitration following focal ischemia in neonatal rat. J Neurochem 70:2516-2525

21. Gelot A, Villapol S, Billette de Villemeur T, Renolleau S, Charriaut-Marlangue C 2009 Astrocytic demise in the developing rat and human brain after hypoxicischemic damage. Dev Neurosci 31:459-470

22. Joly LM, Mucignat V, Mariani J, Plotkine M, Charriaut-Marlangue C 2004 Caspase inhibition after neonatal ischemia in the rat brain. J Cereb Blood Flow Metab 24:124-131

23. Villapol S, Bonnin P, Fau S, Baud O, Renolleau S, Charriaut-Marlangue C 2009 Unilateral blood flow decrease induces bilateral and symmetric responses in the immature brain. Am J Pathol 175:2111-2120

24. Hardeland R, Pandi-Perumal SR, Cardinali DP 2006 Melatonin. Int J Biochem Cell Biol 38:313-316

25. Stolp HB, Ek CJ, Johansson PA, Dziegielewska KM, Bethge N, Wheaton BJ, Potter AM, Saunders NR 2009 Factors involved in inflammation-induced developmental white matter damage. Neurosci Lett 451:232-236

26. Borlongan CV, Yamamoto M, Takei N, Kumazaki M, Ungsuparkorn C, Hida H, Sanberg PR, Nishino H 2000 Glial cell survival is enhanced during melatonininduced neuroprotection against cerebral ischemia. FASEB J 14:1307-1317

27. Sinha K, Degaonkar MN, Jagannathan NR, Gupta YK 2001 Effect of melatonin on ischemia reperfusion injury induced by middle cerebral artery occlusion in rats. Eur J Pharmacol 428:185-192

28. Samantaray S, Das A, Thakore NP, Matzelle DD, Reiter RJ, Ray SK, Banik NL 2009 Therapeutic potential of melatonin in traumatic central nervous system injury. J Pineal Res 47:134-142

29. Wang X, Figueroa BE, Stavrovskaya IG, Zhang Y, Sirianni AC, Zhu S, Day AL, Kristal BS, Friedlander RM 2009 Methazolamide and melatonin inhibit mitochondrial cytochrome $\mathrm{C}$ release and are neuroprotective in experimental models of ischemic injury. Stroke 40:1877-1885

30. Carloni S, Perrone S, Buonocore G, Longini M, Proietti F, Balduini W 2008 Melatonin protects from the long-term consequences of a neonatal hypoxic-ischemic brain injury in rats. J Pineal Res 44:157-164

31. Signorini C, Ciccoli L, Leoncini S, Carloni S, Perrone S, Comporti M, Balduini W, Buonocore G 2009 Free iron, total F-isoprostanes and total F-neuroprostanes in a model of neonatal hypoxic-ischemic encephalopathy: neuroprotective effect of melatonin. J Pineal Res 46:148-154

32. Huang LT, Tiao MM, Tain YL, Chen CC, Hsieh CS 2009 Melatonin ameliorates bile duct ligation-induced systemic oxidative stress and spatial memory deficits in developing rats. Pediatr Res 65:176-180

33. Koh PO 2008 Melatonin regulates nitric oxide synthase expression in ischemic brain injury. J Vet Med Sci 70:747-750

34. Ozdemir D, Uysal N, Gonenc S, Acikgoz O, Sonmez A, Topcu A, Ozdemir N, Duman M, Semin I, Ozkan H 2005 Effect of melatonin on brain oxidative damage induced by traumatic brain injury in immature rats. Physiol Res 54:631-637

35. Haynes RL, Folkerth RD, Trachtenberg FL, Volpe JJ, Kinney HC 2009 Nitrosative stress and inducible nitric oxide synthase expression in periventricular leukomalacia. Acta Neuropathol 118:391-399

36. Husson I, Mesples B, Bac P, Vamecq J, Evrard P, Gressens P 2002 Melatoninergic neuroprotection of the murine periventricular white matter against neonatal excitotoxic challenge. Ann Neurol 51:82-92

37. Welin AK, Svedin P, Lapatto R, Sultan B, Hagberg H, Gressens P, Kjellmer I, Mallard C 2007 Melatonin reduces inflammation and cell death in white matter in the mid-gestation fetal sheep following umbilical cord occlusion. Pediatr Res 61:153158

38. Bouslama M, Renaud J, Olivier P, Fontaine RH, Matrot B, Gressens P, Gallego J 2007 Melatonin prevents learning disorders in brain-lesioned newborn mice. Neuroscience 150:712-719

39. Gressens P, Schwendimann L, Husson I, Sarkozy G, Mocaer E, Vamecq J, Spedding M 2008 Agomelatine, a melatonin receptor agonist with 5-HT(2C) receptor antagonist properties, protects the developing murine white matter against excitotoxicity. Eur J Pharmacol 588:58-63

40. Azzopardi DV, Strohm B, Edwards AD, Dyet L, Halliday HL, Juszczak E, Kapellou O, Levene M, Marlow N, Porter E, Thoresen M, Whitelaw A, Brocklehurst P 2009 Moderate hypothermia to treat perinatal asphyxial encephalopathy. N Engl J Med $361: 1349-1358$ 